\title{
Nord-Mali: les frontières coloniales de l'Azawad
}

\section{Pierre Boilley}

To cite this article: Pierre Boilley (2019): Nord-Mali: les frontières coloniales de l'Azawad, Canadian Journal of African Studies / Revue canadienne des études africaines, DOI: 10.1080/00083968.2019.1667840

To link to this article: https://doi.org/10.1080/00083968.2019.1667840

$$
\text { Published online: } 17 \text { Nov } 2019 .
$$

5 Submit your article to this journal $\square$

Q View related articles $₫$

View Crossmark data $\nearrow$ 


\title{
Nord-Mali: les frontières coloniales de l'Azawad
}

\author{
Pierre Boilley \\ Institut des mondes africains (IMAf), Université Paris 1 Panthéon-Sorbonne, Paris, France
}

\begin{abstract}
RÉSUMÉ
La revendication en 2012 de l'indépendance de l'Azawad a été strictement limitée au seul Mali et à ses frontières septentrionales, d'origine coloniale. Un "État (pan)touareg" regroupant les populations touarègues (Algérie, Mali, Niger, Burkina-Faso, Libye) n'a pas non plus été revendiqué. Cette situation ne peut s'expliquer sans tenir compte des héritages anciens. La définition des territoires impériaux, et des frontières coloniales, connut un processus très différent de ce que suggère le mythe de la conférence de Berlin. Les logiques à l'œuvre témoignent d'une co-construction des frontières avec les populations locales, ou pour le moins le résultat de stratégies croisées de l'administration coloniale et des pouvoirs africains. Les rébellions récurrentes, et le séparatisme récent, ne peuvent donc avoir comme seule explication les tracés frontaliers. II faut intégrer dans l'analyse les histoires locales, et surtout la gestion politique et économique des marges par l'administration française puis par les États indépendants.
\end{abstract}

\begin{abstract}
The claim to independence made by Azawad in 2012 was strictly limited to Mali and its northern borders, which are of colonial origin. No "(pan)Tuareg state" bringing together Tuareg populations (in Algeria, Mali, Niger, Burkina Faso, and Libya) was asserted either. This situation cannot be explained without accounting for ancient legacies. The processes of defining imperial territories and colonial boundaries differ from the myth of the Berlin Conference. The logics at work show a co-construction of borders involving local populations, or at least the dovetailing strategies of the colonial administration and African powers. The tracing of borders cannot therefore operate as a single explanation for recurring rebellions and more recent separatism. It is necessary to incorporate into our analysis local histories and especially the political and economic management of borderlands by the French colonial administration and later independent states.
\end{abstract}

\section{MOTS-CLÉS}

Mali; frontières; Azawad; rébellions; Touaregs

\section{KEYWORDS}

Mali; borders; Azawad; rebellions; Tuaregs

La revendication en 2012 de l'indépendance d'un État de l'Azawad a été strictement et étonnamment limitée au seul Mali et à ses frontières septentrionales, d'origine coloniale. Un "État (pan)touareg" regroupant les populations touarègues des cinq pays les abritant (Algérie, Mali, Niger, Burkina-Faso, Libye) n'a pas non plus été revendiqué. Une telle situation ne peut s'expliquer sans tenir compte des héritages anciens. Les limites intraimpériales qui ont fragmenté les Touaregs ne peuvent ainsi être comprises sans en 
référer aux situations politiques précoloniales qui en ont été le soubassement. De plus, la définition des territoires impériaux, et des frontières coloniales qui en sont les manifestations concrètes, connut un processus très différent de ce que suggère classiquement le mythe de la conférence de Berlin. Les logiques à l'œuvre témoignent plutôt d'une co-construction des frontières avec les populations locales, ou pour le moins le résultat de stratégies croisées de l'administration coloniale et des pouvoirs africains. Les rébellions récurrentes, et le séparatisme récent, ne peuvent donc avoir comme seule explication les tracés frontaliers, intégrés jusque dans la définition d'un espace revendiqué comme indépendant. II faut intégrer dans l'analyse les histoires locales, et surtout la gestion politique et économique des marges par l'administration française puis par les États indépendants.

Le 17 janvier 2012, avec l'attaque de la ville de Ménaka, le MNLA (Mouvement national de libération de l'Azawad) déclenchait une révolte armée au nord du Mali. Après des opérations militaires rapides qui repoussaient les Forces armées maliennes (FAMA) au sud de Tombouctou, ce mouvement déclarait triomphalement le 6 avril l'indépendance de l'Azawad. L'espace revendiqué comprenait tout le nord du pays, coupant le Mali en deux avec l'invention d'une nouvelle limite laissant Mopti au sud, mais respectant strictement les frontières avec la Mauritanie, l'Algérie, le Niger et le Burkina-Faso (cf. Figure 1). À Bamako, on dénonçait aussitôt une "construction politique," l'Azawad n'étant géographiquement qu'un espace peu étendu au nord d'Araouan. Cet argument n'avait évidemment que peu d'intérêt, les espaces nationaux étant tous, de par le monde, des constructions éminemment politiques, mais on oubliait surtout de poser une question plus essentielle: pourquoi les indépendantistes conservaient-ils les frontières maliennes nationales? Pourquoi n'étendre cette petite région qu'aux seules frontières du Mali, et pas au-delà? Par ailleurs, nombre de médias mal informés, notamment occidentaux, tenaient pour évidente l'explication identitaire. N'était-il pas normal qu'un "peuple touareg," éclaté entre cinq pays par le découpage colonial, veuille recouvrer sa liberté sur un territoire qui lui était propre avant l'arrivée des Français? Les frontières coloniales qui avaient divisé les Touaregs entre le Mali, l'Algérie, la Libye, le Niger et le Burkina-Faso n'étaient-elles pas, comme on le savait bien, absurdes et artificielles?

Et de fait, les frontières en Afrique, symboles de l'arbitraire colonial et bornes des territoires impériaux, ont suscité et suscitent de façon récurrente de nombreux débats qui ne sont pas toujours, loin s'en faut, de caractère scientifique. L'idée généralement admise est que ces frontières ont été tracées à la conférence de Berlin, en 1885, à grands coups de crayon traçant des lignes droites sur des cartes globalement vierges. Absurdes, artificielles, divisant les peuples pour mieux les contrôler, elles auraient créé des dommages irréversibles. Confirmées par l'OUA aux indépendances selon le principe adopté de "I'intangibilité des frontières issues de la colonisation," elles seraient à l'origine des nombreux conflits africains depuis un demi-siècle et, pourquoi pas, du sousdéveloppement du continent. Un des cas d'école illustrant ces affirmations pourrait être le partage du Sahara et l'éclatement des populations nomades, à cause de ces lignes découpant un espace auparavant libre et sous domination politique et culturelle des Touaregs, lignes droites et semblant dépourvues de toute signification géopolitique. Quoi de plus aberrant et destructeur que ces limites transperçant un "peuple" qu'on a divisé en cinq colonies, devenues cinq États indépendants, et qui se retrouve marginalisé 


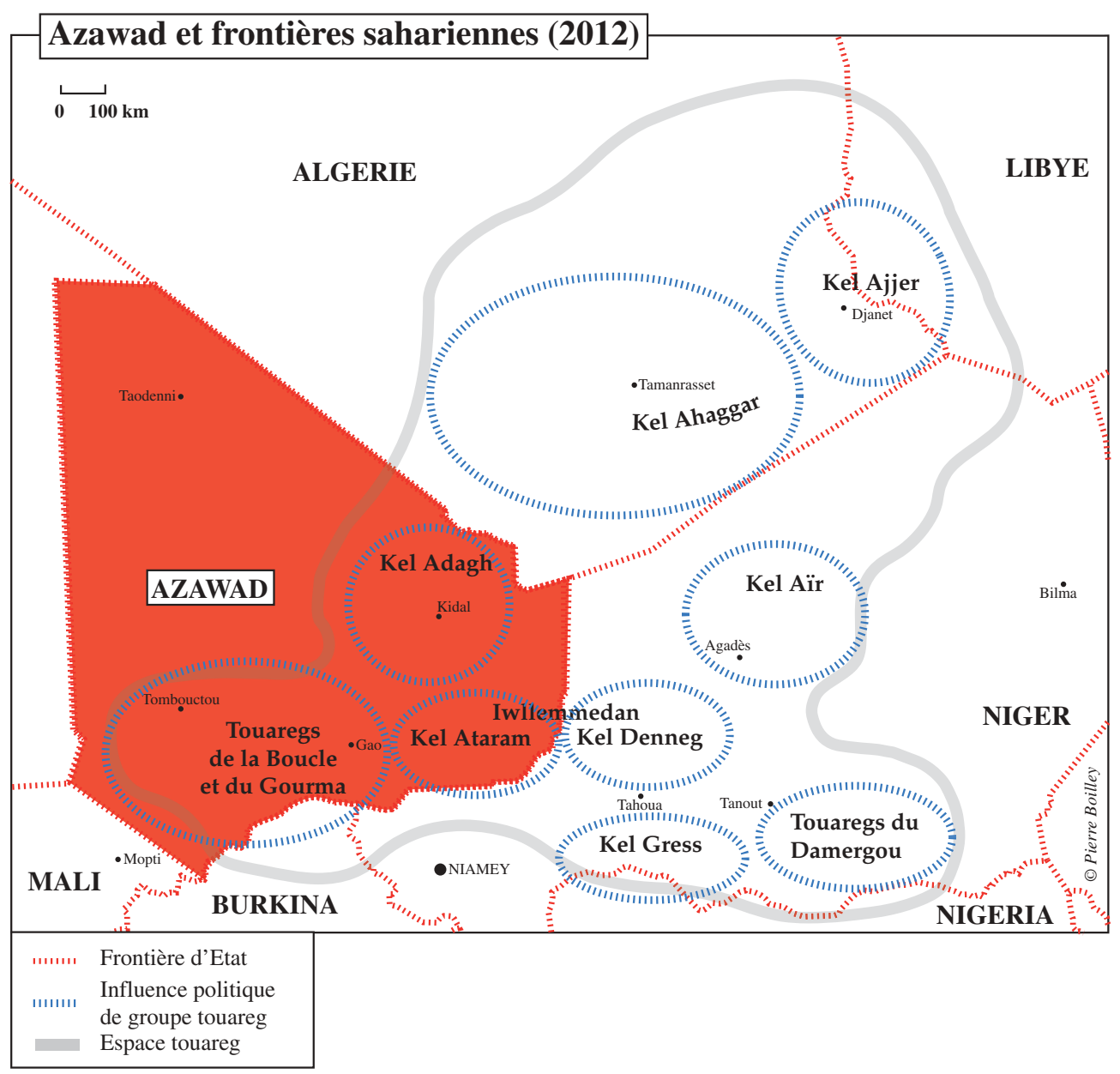

Figure 1. Azawad et frontières sahariennes (2012).

aux confins de ceux-ci? Quoi de plus normal dans ces conditions que des révoltes à répétition ébranlent les États sahéliens, puisqu'une unité identitaire est bafouée? Cet éclatement est ainsi souvent devenu la base des analyses concernant la situation saharienne actuelle, apparaissant comme exemplaire des problèmes de minorités ou "d'ethnicité" en Afrique, mais aussi de l'arbitraire de ces limites "tracées dans les chancelleries européennes." ${ }^{1}$ Quoi de plus apparemment significatif en effet de la "boucherie diplomatique coloniale" ${ }^{2}$ que ces lignes tracées à la règle à travers les espaces sahariens, et de la résistance acharnée des populations concernées pour tenter d'empêcher leur dislocation et retrouver leur unité? N'est-il pas évident que le colonisateur a, au mieux, ignoré les réalités de cet espace qu'il connaissait mal ou, au pire, volontairement divisé - pour mieux régner - les populations touarègues sur lesquelles il voulait étendre sa domination? Mais alors, pourquoi le MNLA n'a-t-il pas cherché à créer un espace beaucoup plus étendu, pan-touareg, sur les cinq pays abritant les populations de ce groupe culturel? Pourquoi avoir utilisé dans un sens restrictif, limitées au seul Mali, ces frontières d'origine coloniale? 
De fait, les analyses trop rapides, qui ne tiennent pas compte ni de l'historicité des situations ni des sources, ne peuvent rendre compte de situations toujours beaucoup plus complexes qu'on ne le pense. Les Touaregs ont-ils connus historiquement une union politique qui justifierait la revendication d'un "État touareg"? Et ces frontières qui matérialisent leur éclatement, en sont-elles la cause ou reflètent-elles une situation antérieure? Pour évaluer les rapports de force qui ont présidé à la construction des frontières, il est impératif de comprendre leur formation, car elles sont "des constructions géopolitiques datées." ${ }^{3}$

\section{Des Touaregs divisés, des histoires éclatées}

Une première interrogation s'impose donc. Avant la conquête, les Touaregs étaient-ils unis? Les voyageurs du XIXe siècle, et les premiers récits des Européens qui ont été en contact avec eux, ${ }^{4}$ obligent à constater qu'il est difficile de répondre par l'affirmative à cette question. Plusieurs pôles politiques (ou ettebel en tamashaq, la langue touarègue) affirmaient en effet leur autorité sur différents espaces du Sahara central. Les Kel Ahaggar au nord, dans le massif de l'Ahaggar, étaient voisins des Kel Ajjer, plus à l'est. Vers le sud, dans ce qui correspond actuellement au nord du Niger, les Kel Aïr, dans le massif de l'Aïr, s'étaient placés sous l'autorité du sultan d'Agadez. Plus au sud encore, les Iwlliminden, séparés en Kel Attaram et Kel Dinnik (ceux de l'ouest et de l'est), avaient chacun leur propre ettebel et leur propre amenokal (le chef d'un pôle politique). Les Kel Ansar, autour de Tombouctou, les Tademekkat, dans la boucle du fleuve Niger, régnaient en maîtres sur l'ouest de l'espace touareg. II faudrait encore citer les Kel Adagh, dans le massif du même nom, les Kel Gress, et d'autres encore (cf. Figure 1). Ces différents pôles étaient en concurrence, voire s'affrontaient dans leur volonté d'assurer leur suprématie sur une région particulière. Ainsi les Kel Adagh étaient-ils dominés par les Kel Ahaggar au nord et les Iwlliminden au sud, qui cherchaient chacun à accroitre leur maîtrise de l'espace pastoral de l'Adagh (actuellement au nord du Mali), riche et bien arrosé. Des tributs en nature ${ }^{5}$ concrétisaient chaque année la soumission des Kel Adagh à ces deux pôles puissants. II est intéressant de voir que cette domination qui pesait sur les Kel Adagh ne prit fin qu'avec l'arrivée des Français. En effet, des stratégies croisées furent mises en œuvre, l'amenokal des Kel Ahaggar, Moussa ag Amastane, cherchant à utiliser les volontés d'expansion de l'Algérie vers le sud incarnées par le colonel Laperrine au détriment de l'Afrique occidentale française, et les Kel Adagh s'alliant avec les autorités de I'AOF pour tenter de gagner leur autonomie. ${ }^{6}$ Après de multiples péripéties qu'il serait trop long de relater ici, une réunion des différents groupes autour du chef de bataillon Bétrix, le 26 décembre 1908, fixa les nouveaux rapports d'autonomie des Kel Adagh avec leurs voisins, montrant ainsi en creux l'âpreté et la conflictualité des rapports entre pôles politiques touaregs. Le compte-rendu de cette réunion, faisant office de traité signé par les parties, précise en effet que "Les Iforas [les Kel Adagh] sont indépendants.... Ils n'ont plus rien à payer à leurs voisins et deviennent enfin les maîtres chez eux"; "Il est entendu que les Hoggars restent en dehors de la question, comme étrangers au pays"; "Les Ouliminden n'ont aucune attache dans l'Adrar." autres faits montrent à l'envie l'absence d'unité des pôles politiques touaregs, avant et au moment de la conquête française. Ainsi, par exemple, lors de la révolte en 1916 de Firhun, amenokal des Iwlliminden, Moussa ag Amastane, loin de le rejoindre dans la 
lutte, prévint le capitaine de la Roche, commandant le secteur Ahaggar ${ }^{8}$ de la rébellion naissante. Pire, lors de la dernière bataille de Firhun contre les Français, le 9 mai 1916 à Anderamboukane, Kel Ahaggar et Kel Adagh participèrent à l'assaut contre lui.....

On ne peut donc voir les populations touarègues du Sahara central, au début du XXe siècle, comme un groupe uni, solidaire dans la résistance à la conquête coloniale, et cherchant à préserver un territoire commun dirigé par une autorité à laquelle tous se référaient. Bien au contraire, chaque pôle politique se considérait comme une entité propre, agissant dans son propre intérêt, suivant sa propre chefferie, en concurrence avec les autres groupes sur le plan territorial et politique, et n'hésitant pas à s'allier avec une force extérieure considérée comme partenaire dans des stratégies conflictuelles. Les Français en ce sens ne divisèrent pas pour régner, mais utilisèrent plutôt les divisions existantes pour mieux s'établir dans l'espace saharien. Les frontières qu'ils mirent ensuite en place, encore simples limites administratives entre colonies du même empire, ne firent en somme qu'entériner ces clivages internes. On peut même montrer qu'elles tinrent compte des réalités locales pour mieux en fixer les tracés.

\section{Frontières coloniales en co-construction, le reflet des divisions}

Les frontières coloniales en Afrique ont été l'objet ces dernières années de nombreux travaux ${ }^{10}$ qui cherchent à en comprendre l'historicité, et les processus mis en œuvre par les puissances coloniales pour les établir. Il apparaît dans de très nombreux cas que ces processus tiennent plus de la co-construction des frontières par l'administration coloniale et les populations ou pouvoirs locaux que d'une imposition par la force de limites qui n'auraient pas été légitimées par d'anciens tracés. Ces derniers, reconnus par les enquêtes effectuées localement par les missions de délimitation, ont été clairement pris en compte pour tracer les frontières. Ces limites ont été créées au cours d'opérations qui ont pris souvent de longues années, a contrario de ce que peut faire penser le mythe de la conférence de Berlin. II n'est besoin d'ailleurs que de se référer au texte ${ }^{11}$ de cette rencontre internationale pour constater qu'aucune frontière n'y a été tracée, ni même que des zones d'influence y aient été pensées. Les seules clauses concernant l'occupation de territoires n'évoquent que les occupations sur les côtes du continent, dans les articles 34 et 35, regroupés sous le titre "Déclaration relative aux conditions essentielles à remplir pour que des occupations nouvelles sur les côtes du continent africain soient considérées comme effectives." ${ }^{12}$ Le cliché de Berlin a néanmoins la vie dure, en dépit des efforts des historiens pour le détruire. ${ }^{13}$

Dans le cas des frontières qui nous intéressent ici, c'est à dire essentiellement celles des dyades ${ }^{14}$ Algérie-Mali, Algérie-Niger et Niger-Mali, il est possible de constater qu'elles proviennent de racines anciennes ou de réalités locales. On a vu plus haut le différend opposant les Kel Adagh et les Kel Ahaggar. La limite qui les a séparés est issue de cette réalité ancienne. Si l'on regarde attentivement le tracé qui délimite les territoires de l'Algérie et du Mali actuel, on peut observer une longue ligne droite partant du point le plus septentrional du nord du Mali et courant à travers le Sahara vers le sud-est. Elle traverse l'erg Iguidi, un espace de sable et de dunes sans puits, puis le Tanezrouft, un reg rocailleux et plat où l'eau est également absente. Ces espaces n'ont jamais été peuplés, mais seulement traversés par les caravanes qui ne s'y attardaient pas. II n'y avait pas avant la colonisation d'autorité territoriale les contrôlant. On peut donc comprendre 
que le caractère rectiligne de cette portion de frontière ne repose effectivement sur aucun tracé ancien. II n'y a eu là pour l'administration coloniale qu'une commodité de délimitation. Il est facile en revanche d'observer que la suite de cette frontière, qui court jusqu'au point de jonction entre l'Algérie, le Mali et le Niger est particulièrement tourmentée (cf. Figure 2). C'est qu'elle a fait l'objet non seulement de discussions entre deux ministères français, celui de l'Intérieur pour l'Algérie, et des Colonies pour I'AOF, mais aussi du partage des parcours de nomadisation entre Touaregs Kel Ahaggar et Kel Adagh. Elle a été concrétisée initialement par la signature, entre le Ministère de I'Intérieur et celui des Colonies, de la convention du 7 Février 1905 de délimitation entre l'Algérie et l'AOF, et prenait la forme suivante:

La délimitation part de la source de l'oued Tin Zaouaten et suit vers l'Ouest la ceinture du bassin du Tilemsi jusqu'à son point le plus septentrional, de là à travers le Tanezrouft occidental elle va couper la route Marabouti-Taoudenni à mi-chemin environ de ces deux points, pour se diriger ensuite directement vers le cap Noun; vers l'Est, la démarcation suit l'Oued Tin Zaouaten jusqu'à sa perte dans le Tanezrouft, qui en laisse la rive nord à l'Algérie et la rive sud à l'Afrique Occidentale Française. Elle se dirige ensuite à peu près à égale distance entre Rhat et la route d'Agadès à Mourzouk. En outre il a été spécifié que la limite indiquée pourra recevoir ultérieurement certaines modifications lorsque les pays qu'elle traverse seront mieux connus. ${ }^{15}$

Cette dernière notification est importante. De fait, la partie est de la frontière fut amendée dans les années suivantes et ces modifications résultèrent des rapports établis par les colonels Laperrine et Ronget. L'administration coloniale a surtout cherché à tenir compte des réalités du terrain, points d'eau, zones de pâturage et répartition des groupes touaregs. Suite à ces rapports, le colonel Laperrine pour

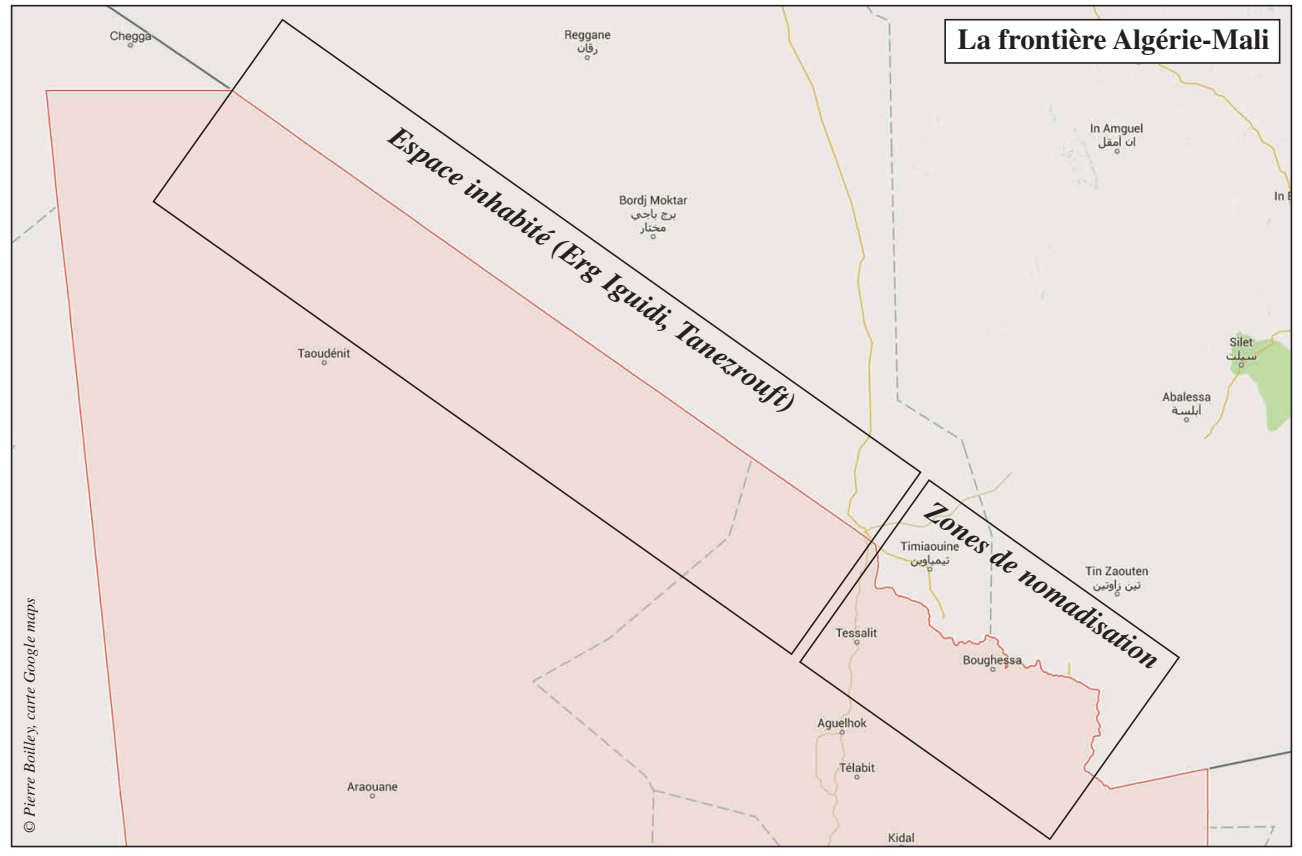

Figure 2. La frontière Algérie-Mali. 
I'Algérie, et le colonel Venel pour l'AOF, négocièrent les modifications de l'Accord de 1905, qui furent intégrées dans la convention de Niamey du 20 juin $1909 .^{16}$ Par celle-ci, il est affirmé que l'Algérie garde les zones de parcours des Touaregs Kel Ajjer et ceux des Touaregs Kel Ahaggar. L'AOF conserve quant à elle les espaces de nomadisation des Kel Adagh. D'autres modifications à ce tracé ont été introduites plus tard avec l'attribution de puits à l'un ou l'autre des territoires, afin de répartir l'eau, vitale pour les groupes nomades. On voit donc bien que pour cette frontière, la logique historique de séparation existante entre Kel Ahaggar et Kel Adagh a été respectée et précisée par une étude fine sur le terrain des parcours nomades et des revendications d'appartenance de chaque groupe.

La frontière entre l'Algérie et le Niger montre une historicité tout aussi intéressante (cf. Figure 3). Les deux conventions du 7 février 1905 et du 20 juin 1909 concernaient aussi les tracés de cette limite. La carte montre une longue ligne droite s'infléchissant à partir des puits d'In Guezzam et d'Assamaka (aujourd'hui encore lieux de postes-frontières) et filant vers l'est-nord-est jusqu'au point de jonction avec la Libye et le Tchad. L'ensemble de cette limite court dans des zones complètement désertiques, sans puits notables outre ceux qui viennent d'être cités. Avant la conquête coloniale, personne ne se souciait de contrôler physiquement cette bande de désert, vide d'hommes. Mais ce long espace était une frontière zone

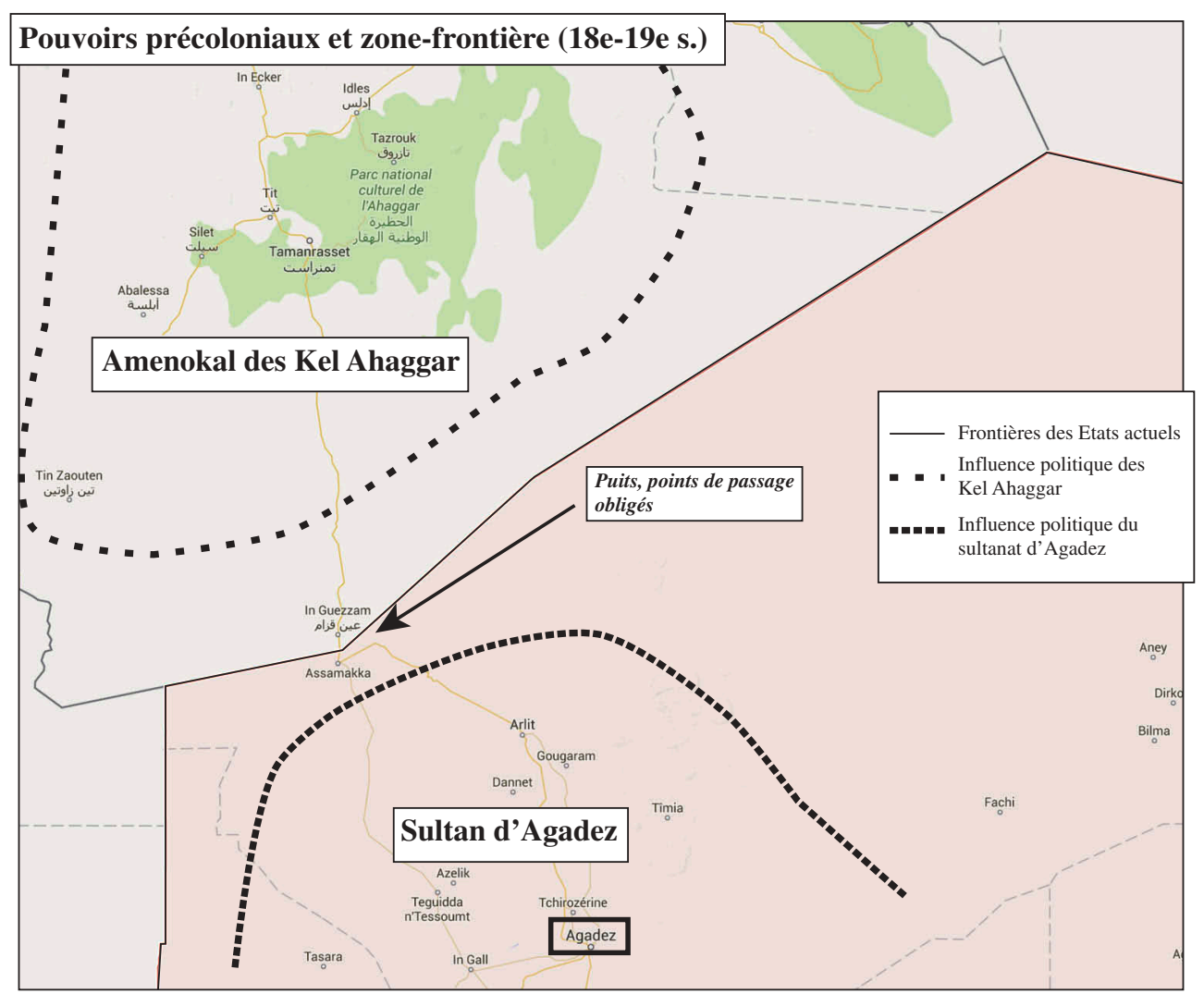

Figure 3. Pouvoirs précoloniaux et zone-frontière $\left(18^{\mathrm{e}}-19^{\mathrm{e}}\right.$ s.). 
séparant le pouvoir de l'amenokal des Kel Ahaggar de celui du sultan d'Agadez, chef des Touaregs Kel Aïr. En témoignent des textes, parfois très anciens, qui évoquent la taxation des voyageurs franchissant cette zone dans un sens ou dans l'autre. Le Livre de l'origine du sultanat de l'Aïr contenu dans les Chroniques d'Agadez, et qui remonterait au XVle siècle, ${ }^{17}$ fait déjà état de ces taxes qui matérialisent le passage entre les territoires dominés par deux pouvoirs différents. Des récits du XIXe siècle viennent confirmer ce fait: "'Plusieurs sources ... permettent d'analyser son fonctionnement à cette époque, notamment Erwin de Bary et El Hadj Ahmed el Fellati, ainsi que les données issues d'un entretien réalisé à la cour du sultan. ${ }^{18}$ Le sultanat d'Agadez perçoit une taxe sur les caravanes entrant dans l'Aïr depuis le nord. Après avoir traversé la zone frontière, les caravanes arrivent dans les premiers villages de l'Aïr, puis à Iférouane. Là, elles rencontrent un représentant du sultan chargé de prélever la taxe. Erwin de Bary arrive à Iférouane le 8 février 1877 avec une caravane de marchands Kel Oui. Le lendemain de son arrivée, il rencontre le représentant du sultan: 'Aujourd'hui l'oukil du sultan d'Agadès, qu'on appelle ici Touraoua, perçoit la redevance due par tous les marchands.' ${ }^{19}$ El Hadj Ahmed el Fellati, qui a traversé cette frontière lors du retour de son premier pèlerinage en $1882,{ }^{20}$ décrit ainsi le fonctionnement de ce système de taxe:

Le sultan d'Aghadès a un oukil, dans le pays d'Agazer, pour la perception des droits sur la frontière. II se nomme actuellement El Hadj Mohamed; il habite généralement Tchintagoda, mais il vient à l'époque des caravanes au printemps, occuper une petite maison qu'il possède à Ifrouen sur la frontière même de l'Aïr. À cette même époque, vient d'Aghadès le Serki n'Touraoua, ou chef des blancs, fonctionnaire chargé du contrôle du commerce. Chaque charge de chameaux [sic] paie une pièce d'étoffe ou de turban. Chaque caravane, à Ifrouen, déballe ses marchandises devant le serki; ce dernier informe les marchands du prix courant de chaque objet tant à Aghadès qu'à Kano.... Quand l'époque des caravanes est terminée, le Serki n'Touraoua rentre à Aghadès rapportant le produit de la douane....."21

On peut remarquer ici que le contrôle ne s'exerce pas sur la frontière, mais dans un dispositif $d$ 'arrière-frontière. La zone frontière étant entièrement désertique au sens strict du terme, le dispositif de contrôle est installé dans les zones habitées. ${ }^{22}$ L'existence de cette zone-frontière, matérialisée par des taxes imposées aux commerçants et caravaniers, est confirmée par de nombreux voyageurs et explorateurs, depuis Ibn Battuta ${ }^{23}$ jusqu'à Henrich Barth. ${ }^{24}$ Lorsque I'administration coloniale décida du tracé, ces récits étaient connus par elle, et furent confirmés par les missions qui enquêtèrent sur le terrain auprès des populations et des autorités africaines. On pourrait enfin en dire autant de la frontière Mali-Niger (cf. Figure 4). Commençant au nord par une ligne droite allant vers le sud, elle commence à s'indenter pour mieux suivre la zone frontière existant entre les Touaregs Iwllimindan qui s'étaient séparés entre Kel Ataram (ceux de l'ouest) et Kel Denneg (ou Dinnik, ceux de l'est). ${ }^{25}$

La logique coloniale était donc de conserver au maximum les limites anciennes afin d'administrer plus facilement les territoires conquis, et de se reposer sur ce qui existait avant la conquête pour mieux servir les intérêts mêlés des colonisateurs et des populations concernées. Ici encore, les processus de délimitation témoignent d'une co-construction de la frontière, basée sur des réalités anciennes. Des lignes remplacent des zones, mais ces zones étaient effectivement d'anciennes frontières. 


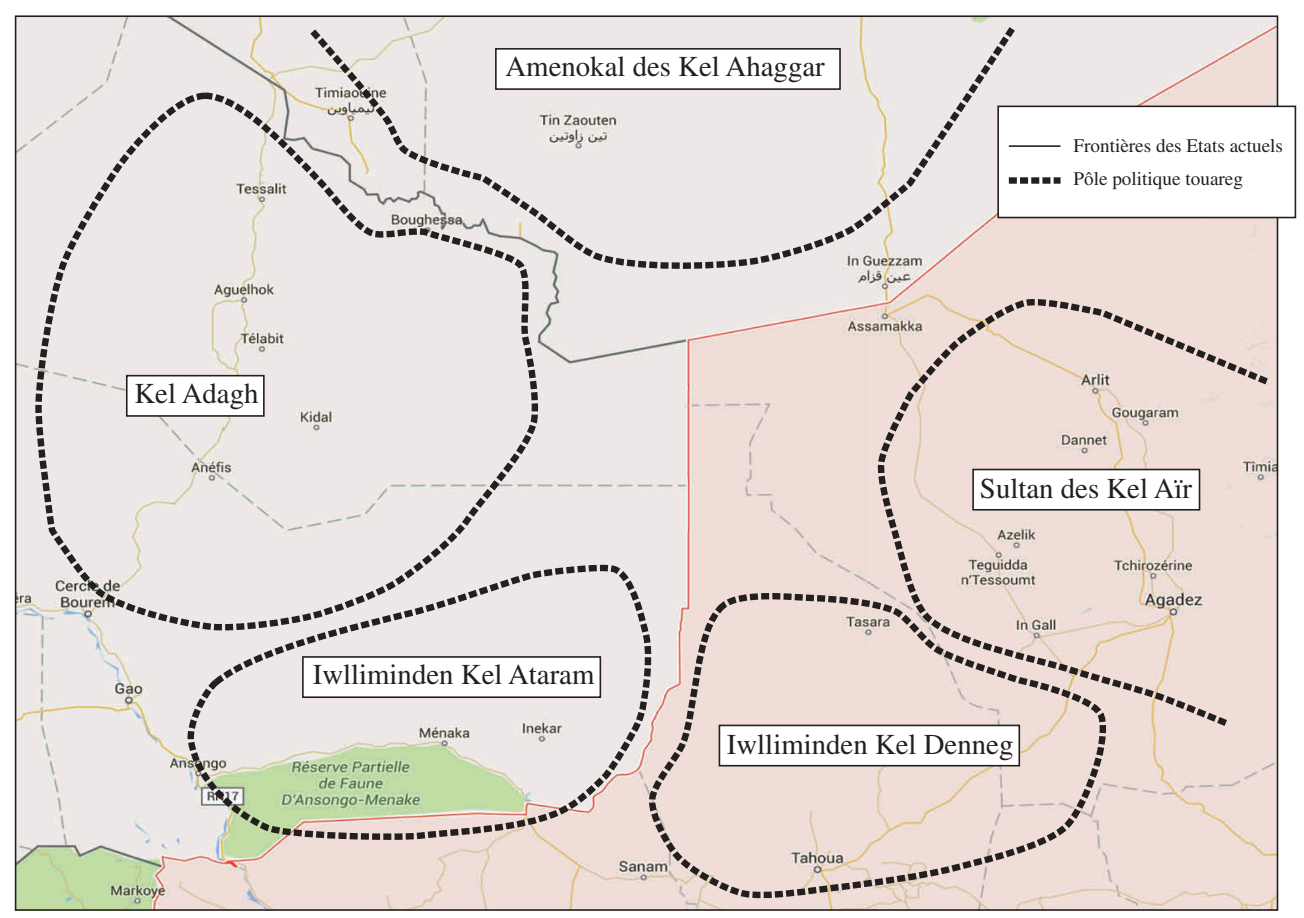

Figure 4. Frontière Mali-Niger.

\section{Frontières coloniales, frontières nationales}

Si les frontières ci-dessus présentées sont à ce point proches de l'existant précolonial et des limites séparant les pouvoirs anciens, comment alors comprendre les séries de rébellions récurrentes au Niger, et surtout au Mali, depuis les indépendances? Faut-il y voir une réaction violente à la transformation de ce qui n'était que des limites administratives intra-impériales françaises en limites étatiques des nouveaux États, plus rigides et moins perméables? Cette étatisation des frontières n'a-t-elle pas consacré la division des populations touarègues qui auparavant pouvaient sans trop de difficultés franchir des bornes gérées par une seule administration coloniale?

Pour répondre à cette question, il faudrait tout d'abord relativiser le changement de nature de ces limites à l'indépendance. Les populations nomades traversaient assez facilement les limites des colonies, mais un grand nombre de rapports de l'administration française montre que ces déplacements étaient pour partie d'entre eux contrôlés, voire gênés par les colonisateurs. Le passage des frontières, notamment depuis le Soudan français ou le Niger vers l'Algérie, où la taxation fiscale était plus légère pour les éleveurs, a été surveillé par les administrateurs du sud de l'Algérie, et il fut assez fréquent que des groupes nomades soient refoulés vers leurs colonies respectives. Certaines fractions, dont les parcours de nomadisation se situaient aux limites de deux circonscriptions administratives ou de deux colonies, jouaient avec les frontières et les administrations différentes, assurant être du Soudan quand les méharistes d'Algérie venaient les contrôler, ou inversement: 
Au cours du mois d'Août, je me suis rendu à Tin Zaouten pour une liaison avec le chef de l'annexe du Hoggar avec qui j'ai réglé la question des campements nomades de cette région. Ceux-ci afin de rester indépendants prétendaient dépendre de Kidal ou du Hoggar suivant qu'ils étaient visités par un officier du Soudan ou de l'Algérie. ${ }^{26}$

Les frontières furent aussi utilisées par des groupes qui s'établissaient à long terme dans des régions très éloignées de leurs parcours d'origine. Recensés par une autre administration que celle où ils vivaient, ils échappaient à tout contrôle, tant des autorités de leur lieu de départ qui ne pouvaient matériellement l'exercer, que de celles de leur lieu de résidence qui ne pouvaient légalement le faire. À l'abri des poursuites, ils aidaient les nomades voisins à dissimuler leur richesse:

On connaît la position particulièrement favorisée des Hoggars. A mille lieux de leur circonscription d'origine, ils ne subissent que le contrôle d'Agadès dont l'activité ne veut évidemment pas s'absorber entièrement à leur sujet.... À l'abri des charges normales de l'administration, ils jouissent en outre d'une totale liberté de mouvement. C'est chez eux aussi que les nomades environnants camouflent leurs troupeaux au moment du recensement. C'est chez eux que se réfugient beaucoup de ressortissants d'AOF en situation irrégulière. Ce sont eux qui indiscutablement alimentent le marché clandestin d'armes. ${ }^{27}$

On pourrait multiplier les exemples de ces passages que l'on pourrait qualifier de clandestins mais qui n'étaient pas nécessairement considérés comme tels par les nomades. Les réactions de l'administration coloniales furent aussi variées:

Le moyen le plus fréquemment employé fut la reconduite manu militari, dans des opérations dites de "refoulement," des groupes nomades découverts hors des zones qui leur avaient été reconnues par l'administration. Une fois repérés, les campements fautifs, éloignés les uns des autres, devaient souvent être "rabattus" ${ }^{28}$ par des partisans vers un lieu de regroupement. Les goumiers de la subdivision, ou ces mêmes partisans, les prenant en charge, les escortaient alors jusqu'à leurs terrains de parcours propres. Des amendes étaient distribuées. $^{29}$

Des permis de nomadisation ont aussi été distribués, des "assignations à résidence" de groupes entiers régulièrement prononcés. Mais les administrateurs ont fréquemment reconnu dans leurs rapports la difficulté, voire l'inanité de leurs efforts.

Les indépendances ne changèrent pas grand-chose à cet état de fait. Les nouveaux États africains, encore moins au fait des réalités nomades que l'administration coloniale, cherchèrent bien à contenir les éleveurs dans les frontières nationales. Ce contrôle passa notamment, au Mali, par l'établissement d'un réseau de coopératives d'éleveurs où ces derniers étaient obligés de commercialiser leurs produits au lieu de les exporter dans les pays voisins, notamment en Algérie, par un commerce caravanier qui fut interdit. Les sociétés d'État, SOMIEX (Société malienne d'importation et d'exportation), OPAM (Office des produits agricoles du Mali), étaient seules habilitées à acheter les productions destinées à l'exportation, et en échange proposaient aux nomades les produits dont ils avaient besoin (guinée, percale, cretonne, céréales).

En pratique, cette nouvelle organisation n'atteignit pas le succès que l'administration en attendait. Un contrôle frontalier étanche était impossible à mettre en place, et la proportion du trafic contrôlé fut mineure:

D'une manière générale plus de $60 \%$ du cheptel exporté de l'Adrar ne passe pas par les services de douanes. L'essentiel des recettes douanières du bureau de Tessalit s'opère d'une 
part sur les marchandises (dattes, tapis, tabacs), d'autre part sur le cheptel qui passe par les véhicules algériens. ${ }^{30}$

II en fut de même pour le Niger et l'Algérie. La contrainte que représentaient les frontières fut certainement plus importante, mais les systèmes économiques et politiques différents entre l'Algérie (socialiste et planifiée), le Mali et le Niger offrirent en revanche de nombreuses et nouvelles opportunités de commerce - qui a pu être qualifié de "trafic illicite." Cette fraude - mot qui fut à l'origine du néologisme touareg "afrod" - utilisait par exemple la revente de produits algériens soutenus par l'État dans les territoires nigérien et maliens. Semoule, sucre, thé, macaroni, essence, cigarettes, furent les produits passant les frontières, et jusqu'au lait algérien en poudre, dont la marque donna son nom au "commerce Lahda." Les pièces détachées de voiture ou de camion, l'alcool, difficiles à trouver en Algérie, faisaient le chemin inverse, du Sahel vers le nord. La porosité des frontières fut ainsi tout autant réelle après l'indépendance qu'à l'époque coloniale.

\section{Des frontières à l'épreuve des conflits}

Il est donc nécessaire de chercher d'autres explications aux rébellions touarègues, d'autres interprétations qui ne concernent pas les frontières. On ne pourra ici développer l'histoire de ces rébellions, dont la première éclata au Mali en 1963-1964, et dont les suivantes eurent lieu dans la décennie 1990 au Niger et au Mali, puis en 2006, 2009, et finalement à partir de 2012 au seul Mali. Mais il est intéressant d'en comprendre la teneur au regard de la responsabilité des frontières. En 1990, au Niger et au Mali, les revendications des mouvements rebelles ont été exclusivement intégratrices, revendiquant une meilleure incorporation des Touaregs dans le développement économique et politique de leurs États respectifs. Au moment des accords de Tamanrasset, signés le 6 janvier 1991 par le Mouvement populaire de l'Azawad (MPA, malien), il est en effet demandé la "création de 6 postes ministériels à confier aux ressortissants des 2 régions" (point $\mathrm{n}^{\circ} 4$ ), ainsi que "l'intégration et la prise en charge de nos combattants par le Ministère de la Défense" (point $n^{\circ} 6$ ). Le volet économique des revendications est destiné à rattraper le retard pris par le nord du Mali en matière de développement, comme "la résolution du problème de l'emploi" (point $\mathrm{n}^{\circ} 8$ ), la "réalisation du barrage du Tossaye" (point $n^{\circ} 17$ ), l'amélioration des aspects fiscaux (points $n^{\circ} 9$ et 19) ou bancaires (création de banques agro-pastorales: point $n^{\circ} 13$ ), la promotion de la scolarisation (points $n^{\circ} 10$ et 11 ) et de l'assistance sanitaire (point $n^{\circ} 12$ ), le désenclavement de la région par la construction de routes (point $n^{\circ} 16$ ) ou d'aéroports (point $n^{\circ} 14$ ), et sa mise en valeur par la réalisation de projets agro-pastoraux (point $n^{\circ} 15$ ). $D^{\prime}$ autres points encore montrent à l'évidence le désir d'une meilleure intégration au reste du pays. ${ }^{31}$ II en est de même dans les points du traité suivant, dit "Pacte national," ${ }^{32}$ signé par le Mali et les Mouvements et fronts unifiés de l'Azawad (MFUA), le 11 avril 1992. Au Niger, des revendications équivalentes aboutirent à la signature d'un "Accord établissant une paix définitive entre le Gouvernement de la République du Niger et l'Organisation de la Résistance Armée," ${ }^{\prime 33}$ paraphé à Ouagadougou le 15 avril 1995 et confirmé à Niamey le 24 avril 1995. 
Notons que ces deux rébellions concomitantes ne firent pas jonction entre elles. Les rebelles et les mouvements de libération furent maliens ou nigériens, et les accords de même. Pendant ces révoltes, les frontières étatiques ne furent jamais remises en cause, et il ne fut à aucun moment question d'une revendication portant sur la création d'un État touareg. On retrouvait là les histoires parallèles des différents groupes touaregs, dont on a déjà vu plus haut l'absence d'unité précoloniale. Les Touaregs algériens, Kel Ahaggar et Kel Ajjer, n'avaient pas cherché à s'organiser avec les autres groupes plus méridionaux pour s'opposer à la conquête coloniale, et se firent vaincre chacun de leur côté par les Français. Ces derniers leur imposèrent une administration différente. Intégrés à l'Algérie, ils n'étaient pas gérés par le gouverneur général de l'Afrique occidentale française, comme les Touaregs du Soudan français et du Niger. Ils furent administrés à l'indépendance par un État algérien mieux accepté par les nomades qui s'en sentaient plus proches culturellement qu'avec les pouvoirs "noirs" du Sahel. Vivant aux confins sahariens, mais mieux intégrés, ils ne se lancèrent pas dans les rébellions que connurent les groupes du sud. Ces destins différents sont certainement une des bases de l'absence de revendications d'un État pan-touareg.

En revanche, les Touaregs du Mali et du Niger connurent une forte marginalisation politique et économique et furent en butte à des États centraux les soupçonnant de séparatisme. Cette marginalisation n'était pas nouvelle. Pendant la colonisation, les espaces septentrionaux du Soudan français et du Niger, considérés comme peu utiles économiquement, furent globalement négligés par l'administration. Peu d'infrastructures furent mises en place, à l'exception de pistes, de services médicaux embryonnaires, et d'une scolarisation des enfants nomades très tardive puisque les premières écoles, et en très petit nombre, ne furent fondées qu'au tournant des années 1940 et 1950. On ne chercha pas à transformer les nomades, l'administration coloniale préférant ne pas donner de prétexte à des révoltes toujours possible. Les États indépendants héritèrent de cette mise à l'écart, ainsi que d'une méfiance à l'égard de ces populations considérées comme turbulentes et promptes à se rebeller. La rébellion de 1963-1964, qui fut durement réprimée par l'armée malienne, renforça encore les préventions que pouvait avoir les États contre les Touaregs, particulièrement au Mali mais aussi au Niger, quoique dans une moindre mesure. Le nord du pays, sous administration devenue civile à la fin de la période coloniale, fut remilitarisé après l'indépendance, et le resta jusqu'à la rébellion de 1990. En ce sens, on peut parler d'une marginalisation héritée - mais celle-ci fut encore plus dure après les indépendances. Les revendications des rebelles en 1990 sont claires, et témoignent de la négligence de l'État à l'égard de son nord, sans guère de politique de développement, sans intégration des Touaregs dans les corps en uniforme de l'État, sans véritable représentation politique dans les organes de gouvernement. Le Pacte national a tenté de remédier à cet état de fait, mais les engagements ayant été peu ou mal respectés, les promesses faiblement tenues, le développement ou la mise en place d'infrastructures telles que des routes se faisant attendre, des révoltes nouvelles, de moindre importance, éclatèrent au Mali, notamment en 2006 et 2009.

Dans l'espoir d'améliorer leur situation, les Touaregs tentèrent aussi des actions politiques, notamment lorsque des associations étudiantes cherchèrent à fusionner et créer un parti politique, le MNA (Mouvement national de l'Azawad), à la fin de l'année 2010. Mais l'État réagit en arrêtant immédiatement les deux leaders de ce mouvement. “La tentative légaliste échoue: le MNA n'est pas autorisé par les autorités maliennes et 
les associations impliquées sont interdites de réunions." ${ }^{34}$ La radicalisation et la clandestinité qui en résultèrent aboutirent, de guerre lasse, à la recréation d'un mouvement armé. La rébellion qui s'ensuivit et éclata en 2012 au Mali est éclairante à plus d'un titre. Le Mouvement national de libération de l'Azawad (MNLA) ${ }^{35}$ qui la mena à partir du 17 janvier 2012, afficha dès le début des hostilités une tonalité radicalement indépendantiste. Fortement armée, suite notamment au pillage des arsenaux libyens après la chute de Khaddafi et au retour vers le Mali des Touaregs incorporés dans l'armée libyenne, la rébellion du MNLA conquit tout le nord du Mali en moins de trois mois. Après la chute de Tombouctou le $1^{\text {er }}$ avril 2012, le mouvement déclara unilatéralement le 6 avril l'indépendance de l'Azawad, nom donné au territoire revendiqué. Or, on peut observer, comme on l'a dit plus haut, que l'Azawad "indépendant" est strictement contenu dans les frontières étatiques maliennes. Les frontières avec la Mauritanie, I'Algérie et le Niger ne sont en aucun cas remises en cause. Seule une frontière méridionale est établie au nord de Mopti, séparant l'Azawad du Mali du sud. Qui plus est, cette nouvelle frontière revendiquée se calque sur les limites de cercles administratifs, elles-mêmes issues des cercles coloniaux. Alors même qu'un nouvel État, majoritairement touareg, est proclamé au Mali, aucune revendication d'une extension aux régions touarègues voisines, algériennes ou nigériennes, n'est avancée. L'État de l'Azawad, qui n'a été reconnu par aucun État de la communauté internationale, n'a pas mis en avant la réunion des Touaregs dans un espace commun. On ne peut que constater l'étonnante résistance de ces frontières internationales issues de la colonisation, alors même qu'un conflit d'importance, aggravé par l'irruption des salafistes armés et de la France lançant contre eux son opération Serval, semblait pouvoir les remettre en cause....

\section{De la responsabilité des frontières à celle de l'État}

Les frontières étudiées ici, séparant l'Algérie, le Niger et le Mali, sont donc loin d'être les séparations "arbitraires" ou "artificielles" que l'on évoque généralement. Créées par les Français dans les premières années de la colonisation, elles s'établirent sur un substrat politique existant qui n'a pas écartelé les populations touarègues, mais a plutôt entériné leurs divisions entre pôles politiques concurrents. Les processus de délimitation mirent en œuvre enquêtes de terrain, missions de reconnaissance et remontées des réalités locales, dans le souci de conserver au mieux, pour plus facilement dominer et administrer, les zones-frontières anciennes datant souvent de plusieurs siècles.

Mais la pertinence de ces segments de frontière ne préjuge toutefois pas des difficultés ultérieures. Ainsi, pour le Mali, si les frontières avec l'Algérie, la Mauritanie et le Niger peuvent être expliquées par la remontée de traces frontalières anciennes, qui vont jusqu'à être respectées dans la revendication d'un État indépendant tel que l'Azawad, la réunion de ces différents segments, comprenant les frontières avec le Burkina Faso, la Côte d'Ivoire, la Guinée et le Sénégal, détermine un espace "national," qui n'a pour sa part rien d'historique. Même si le Mali, dans son "roman national," établit une filiation historique avec l'empire du Mali des XIIle et XIVe siècles, il ne s'agit que d'un imaginaire, la solution de continuité entre cette construction politique et l'État contemporain étant béante. II en résulte que ce sont les espaces nationaux qui pourraient poser des problèmes, non leurs limites. Cependant, il est aussi possible de montrer que les 
territoires de ces États ne sont pas plus artificiels que celui des autres États de la planète, et n'abrite pas des populations plus hétérogènes que beaucoup d'entre eux.

De fait, au regard des rébellions touarègues, c'est bien la marginalisation politique et économique d'une population qui est en cause. Les rébellions n'ont pas cherché la rectification ou la reconstruction des frontières, mais ont mis en évidence une situation désastreuse imposée aux populations nomades maliennes et nigériennes. En ce sens, il faut regarder au-delà des frontières, et même du territoire, pour comprendre et analyser ces mouvements de révolte. Lorsqu'un pouvoir national ne gère pas équitablement les différentes régions qui composent le pays, lorsque les ressources de l'État sont détournées ou inéquitablement redistribuées, lorsque des réseaux de clientèle basées sur le lignage ou le cousinage priment, des régions entières sont privées de développement et d'infrastructures. La responsabilité des conflits sahéliens actuels n'est pas à attribuer aux frontières, mais bien aux conséquences désastreuses de la gestion de territoires hétérogènes par les États et leurs élites dominantes.

\section{Notes}

1. A. Benmessaoud Tredano, "Intangibilité des frontières coloniales et espace étatique en Afrique," Bibliothèque africaine et malgache, 47, 1989: 16.

2. H. Deschamps, "La corne de l'Afrique, peuples et frontières," RFEPA, 54, oct. 1978: 25-42.

3. Foucher M., Fronts et frontières. Un tour du monde géopolitique, Paris, Fayard, 1991: 43.

4. Henri Duveyrier, Heinrich Barth, It. Cortier, etc....

5. "Désireux, à cause de leur relative richesse de vivre en paix avec leurs turbulents voisins, les Iforas, peuplade très douce de caractère et qui ne sont pas considérés par les Imochars comme de même sang qu'eux, ont payé des tributs aux Oulminden et aux Hoggar." R. Arnaud, Les nomades de l'Adrar sous la domination française, 1917, Archives du Mali, Fonds anciens 1D27: 5. Cortier parle d'un "tribut de tentes et de nattes que les Ifor'ass payaient jadis aux loulliminden et aux Ahaggar." Lt Cortier, D'une rive à l'autre du Sahara, Paris, Larose, 1908: 280.

6. Boilley 1999a: 86-97.

7. Convention Iforas - Ouliminden - Kountas, 10 février 1909, ANM, Bamako, 1N114, Fonds anciens.

8. A. Richer, les Oulliminden, Paris, Larose, 1924: 332 (note de bas de page).

9. Rapport du Capitaine Loyer sur l'affaire d'Anderamboukane du 9 juin 1916, SHAT, AOF, Soudan 9, dossier 1, pièce 28 .

10. Citons notamment les travaux effectués par le réseau Aborne (African Borderlands Research Network, http://www.aborne.org/), ou encore ceux de l'équipe Frontafrique (http://www. frontafrique.org/).

11. Aisé à trouver, on peut notamment le lire sur http://mjp.univ-perp.fr/traites/1885berlin.htm.

12. "Article 34. La Puissance qui, dorénavant, prendra possession d'un territoire sur les côtes du Continent africain situé en dehors de ses possessions actuelles, ou qui, n'en ayant pas eu jusque-là, viendrait à en acquérir, et de même la Puissance qui y assumera un protectorat, accompagnera l'acte respectif d'une notification adressée aux autres Puissances signataires du présent Acte, afin de les mettre à même de faire valoir, s'il y a lieu, leurs réclamations." "Article 35. Les Puissances signataires du présent Acte reconnaissent l'obligation d'assurer, dans les territoires occupés par elles, sur les côtes du continent africain, l'existence d'une autorité suffisante pour faire respecter les droits acquis et, le cas échéant, la liberté du commerce et du transit dans les conditions où elle serait stipulée...." Acte général de la conférence de Berlin de 1885, 26 février 1885.

13. Voir notamment H. Brunschwig, Le partage de l'Afrique noire, Paris, Flammarion, 1971, 186 p. 
14. Terme proposé par Michel Foucher pour qualifier "une frontière commune à deux États contigus" (Foucher 1991, 15).

15. Bulletin du Comité de I'Afrique Française, ${ }^{\circ} 1$, janvier 1907: 24-25.

16. I. Brownlie et I. Burns, African Boundaries: A Legal and Diplomatic Encyclopaedia, Londres, Hurst \& Co., 1979, 1355 p.

17. Y. Urvoy, "Chronique d'Agadez," Journal de la Société des africanistes, IV, 1934, 145-77.

18. Entretien réalisé par Camille Lefebvre à Agadez, à la cour du sultan Ibrahim Oumarou le 28 avril 2002.

19. Erwin de Bary, Le dernier rapport d'un européen sur Ghât et les Touareg de l'Aïr, traduit et annoté par H. Schirmer, Paris, Fischbacher, 1898, p. 113.

20. A.S. Kanya-Forstner et Paul Lovejoy, Pilgrims, Interpreters and Agents: French Reconnaissance Reports on the Sokoto Caliphate and Borno, 1891-1895, Madison, African Studies Program University of Wisconsin-Madison, 1997, p. 2.

21. El Hadj Ahmed el Fellati, in Kanya-Forstner et Lovejoy, op. cit., 1997, p. 73.

22. C. Lefebvre, Territoires et frontières. Du Soudan central à la République du Niger, 1800-1964, Thèse de doctorat, Université Paris 1 Panthéon-Sorbonne, dir. P. Boilley, 2008, pp. 82-83.

23. Ibn Battuta, in Voyageurs arabes, Ibn Fadlân, Ibn Jubayr, Ibn Battuta, texte traduit et annoté par P. Charles Dominique, Paris, Gallimard, 1995, p. 1048.

24. H. Barth, 1965, Voyages et découvertes dans l'Afrique septentrionale et centrale pendant les années années 1849 à 1855, traduction de l'allemand par Paul Ithier, Paris, A. Bohné, 4 volumes, 1863, tome 1, 370 p., tome 2, 318 p., tome 3, 337 p., tome 4, 304 p.

25. C. Grémont, Les Touaregs Iwellemmedan (1647-1896). Un ensemble politique de la Boucle du Niger, Paris, Karthala, 2010, 557 p.

26. Rapport politique, 3e Trimestre 1933, Capitaine de Saint-Maur, Cercle de Tombouctou Subdivision Kidal. ANM, 1E24, Fonds récents, Bamako Rapports politiques.

27. Annexe $n^{\circ} 5$ au rapport de tournée $n^{\circ} 5 / K$ du 16 septembre 1944 , le lieutenant Lagarde commandant la subdivision de Kidal, Cercle de Gao. ANM, 1E24, Fonds récents, Bamako, Rapports de tournée 1944-49.

28. Rapport de tournée $N^{\circ}$ 6-Ki du 22 septembre au 16 octobre 1943, Lieutenant Lagarde, commandant la subdivision de Kidal. Cercle de Gao, Subdivision de Kidal. ANM, 1E24, Fonds récents, Bamako. Rapports politiques et rapports de tournées.

29. P. Boilley, Les Touaregs Kel Adagh. Dépendances et révoltes: du Soudan français au Mali contemporain, Paris, Karthala, coll. Hommes et sociétés, 1999a, p. 228.

30. E. Ag Foni, L'impact socio-économique de la sécheresse dans le cercle de Kidal, Brême, Borda, 1979, pp. 81-82.

31. "Procès-verbal de la Rencontre entre délégations à Tamanrasset du 10 au 12 octobre 1990." Archives personnelles.

32. Voir notamment le texte à l'adresse (consultée le 22 mai 2016): http://peacemaker.un.org/sites/ peacemaker.un.org/files/ML_920411_PacteNationalGouvMaliAzawad.pdf.

33. Voir notamment le texte à l'adresse (consultée le 22 mai 2016):http://peacemaker.un.org/ sites/peacemaker.un.org/files/NE_950415_AccordPaixDefinitif.pdf.

34. Sur la création du MNA puis du MNLA, voir l'article: D. Chebli, "La révolte en héritage. Militantisme en famille et fragmentation au Nord-Mali (MNLA)," Cahiers d'études africaines, 2019/2, n²34, 453-81.

35. Le MNLA ne se voulait pas exclusivement touareg et se posait en représentant de l'ensemble des communautés vivant au nord du Mali. En pratique, peu de Songhays et de Peuls rejoignirent le mouvement, et une faible proportion seulement des populations arabes y participa.

\section{À propos de l'auteur}

Pierre Boilley est professeur d'Histoire contemporaine de l'Afrique à l'Université Paris 1 Panthéon Sorbonne, et membre de l'Institut des mondes africains (IMAf), unité mixte CNRS. Ses spécialités et enseignements concernent l'État en Afrique, et particulièrement les identités culturelles, le 
sentiment national, les frontières africaines et les révoltes et rébellions sahariennes et sahéliennes. II a publié, entre autres ouvrages, Les Touaregs Kel Adagh. Dépendances et révoltes: du Soudan français au Mali contemporain (Paris, Karthala, coll. Hommes et sociétés, 1999, réed. 20121), Amidou Mariko, Mémoires d'un crocodile. Du sujet français au citoyen malien (Bamako, Editions Donniya, 2001), Nomades et commandants. Administration coloniale et sociétés nomades dans I'ancienne AOF (Paris, Karthala, collection Hommes et sociétés, 1993).

\section{Déclaration}

Aucun conflit d'intérêt potentiel n'a été rapporté par l'auteur.

\section{Références}

Barth, H. 1863. Voyages et découvertes dans l'Afrique septentrionale et centrale pendant les années 1849 à 1855. Traduction de l'allemand par Paul Ithier. Paris: A. Bohné. 4 volumes, tome 1, 370 p., tome 2, 318 p., tome 3, 337 p., tome 4, 304 p.

Barth, H. 1965. Travels and Discoveries in North and Central Africa Being a Journal of an Expedition 1849-1855. Centenary Edition in Three Volumes. Londres: Franck Cass. vol. 1, 657 p., vol. 2, 709 p., vol. 3, 800 p.

Benmessaoud Tredano, A. 1989. "Intangibilité des frontières coloniales et espace étatique en Afrique." Bibliothèque africaine et malgache 47: 16.

Boilley, P. 1999a. Les Touaregs Kel Adagh. Dépendances et révoltes: du Soudan français au Mali contemporain, 644. Paris: Karthala, coll. Hommes et sociétés.

Brownlie, I., and I. Burns. 1979. African Boundaries: A Legal and Diplomatic Encyclopaedia, 1355. Londres: Hurst \& Co. Publishers.

Brunschwig, H. 1971. Le partage de l'Afrique noire, 186. Paris: Flammarion.

Charles Dominique, P. 1995. Voyageurs arabes, Ibn Fadlân, Ibn Jubayr, Ibn Battuta, 1409. texte traduit et annoté par. Paris: Gallimard.

Chebli, D. 2019. "La révolte en héritage. Militantisme en famille et fragmentation au Nord-Mali (MNLA)." Cahiers d'études africaines 234 (2): 453-481. doi: 10.4000/etudesafricaines.25913.

de Bary, E. 1898. Le dernier rapport d'un européen sur Ghât et les Touareg de l'Aïr, 221. traduit et annoté par Henri Schirmer. Paris: Fischbacher.

Foucher, M. 1991. Fronts et frontières. Un tour du monde géopolitique, 691. Paris: Fayard.

Grémont, C. 2010. Les Touaregs Iwellemmedan (1647-1896). Un ensemble politique de la Boucle du Niger, 557. Paris: Karthala.

Kanya-Forstner, S., and L. Paul. 1997. Pilgrims, Interpreters and Agents: French Reconnaissance Reports on the Sokoto Caliphate and Borno, 1891-1895, 189. Madison: African Studies Program University of Wisconsin-Madison.

Lefebvre, C. 2008. "Territoires et frontières. Du Soudan central à la République du Niger, 18001964." Thèse de doctorat, 501 p. (+ annexes). Université Paris1 Panthéon-Sorbonne, dir. P. Boilley.

Urvoy, Y. 1934. “Chronique d'Agadez." Journal de la Société des africanistes IV: 145-177. 